\title{
„Für bestimmte Anwendungsgebiete best geeignete Werkstoffe...finden"
}

\section{Zur Praxis der Forschung an Ersatzstoffen für Metalle in den deutschen Autarkie-Phasen des 20. Jahrhunderts}

\author{
Günther Luxbacher
}

\begin{abstract}
"Detecting the Most Suitable Materials for Certain Applications". The Practice of Substitute Metals Research in the Phases of German Autarky during the $20^{\text {th }}$ century

The focus of this essay is on substitute materials, particularly the research on substitute metals. The two periods of autarky in Germany were of primary influence on this field. Engineers and construction engineers proved most important in this context despite inter-institutional cooperation between the disciplines of metals research, materials testing, and technical mechanics. Engineers had to solve construction problems respecting the materials used and to analyse components in terms of economic efficiency. However, the research on substitute metals not only meant the substitution of one substance for another. It also implied economizing and recycling scarce materials. These activities required tacit knowledge. Engineers succeeded to some degree but the German war economy paid a high price for it. Each technical system demanded a case-specific approach. Typically a loss of effectiveness, reliability and durability of the products containing substitutes had to be taken into account.
\end{abstract}

Keywords: History of technology, war economy, substitute materials, metal research, knowledge by experience

Schlüsse/wörter: Technikgeschichte, Kriegswirtschaft, Ersatzstoffe, Metallforschung, Erfahrungswissen

Die Frage der Versorgung moderner Gesellschaften mit industriellen Rohstoffen zählt zu den grundlegenden, wenngleich oft vernachlässigten Themen der neueren Geschichte (Siegenthaler 1990, Reith 1993, 2001). Seit der Jahrtausendwende hat das Thema der Rohstoffsicherheit jedoch verstärkt Konjunktur (Bundesverband der Deutschen Industrie 2007, Anonym 2006). So gründete das Bundesministerium für Wirtschaft 2005 für Roh- und Werkstofffragen die Deutsche Materialeffizienzagentur (DEMEA) nach dem Muster der 2000 geschaffenen Deutschen Energie-Agentur (DENA) sowie 2010 die Deutsche Rohstoffagentur bei der Bundesanstalt für Geowissenschaften und Rohstoffe. 
Besondere Aufmerksamkeit verdient die industrielle Rohstoffversorgung in Deutschland in den beiden Perioden relativer Autarkie von 1914 bis 1919, also der Zeit des Ersten Weltkriegs einschließlich der alliierten Blockade, sowie der Zeit der nationalsozialistischen Autarkiepolitik vor und während des Zweiten Weltkriegs von 1933 bis 1945. Auf diese historischen Zeiträume sowie auf industrielle Roh- und Werkstoffe, insbesondere Eisen und Stahl sowie Nichteisen-Metalle konzentriert sich dieser Beitrag.

Bereits die Warenkunde wie die Betriebswirtschaftslehre um 1900 sahen in den gewerblichen Roh- und Werkstoffen einen aus technisch-ökonomischer Sicht systematisch zu untersuchenden Gegenstand (Luxbacher 2001). So führte Andreas Voigt, Inhaber des volkswirtschaftlichen Lehrstuhls an der Universität Frankfurt a. Main, die Begriffe der „Stoffökonomie“, der „Stoffersparnis" und des "Nutzungsgrads der Stoffe“ in seine „Technischen Ökonomik“ als eigene Kategorien ein (Voigt 1912: 257, Zachmannn 1995). Er war damit einer der ersten, wenn nicht gar der erste Wissenschaftler, der die Frage von Wirkungsgrad und Wertgrad unter den Aspekten der Technologie, Werkstoff und Rohstoffpreisen analysierte. Gerade in Deutschland war die Kluft zwischen hohem, industriell bedingtem Rohstoffverbrauch bei gleichzeitig großer Importabhängigkeit besonders deutlich. Es ist daher davon auszugehen, dass der Energie- und Stoffwirtschaft in Deutschland ein hoher Stellenwert zukam. Die Problematik beschäftigte jedoch auch Deutschlands industrielle Konkurrenten. Der englische Außenminister Samuel Hoare unterschied 1935 zwischen den „haves“ und den „have nots“, also den Ländern mit und jenen ohne weitgehender Selbstversorgungsmöglichkeit (Bauer 1939: 9-15, Hildebrand 1969: 371). Auch Werner Sombart hat auf die Bedeutung der Sicherung des Rohstoffbezugs für die modernen imperialen Industriestaaten des 19. Jahrhunderts hingewiesen, der oft Vorrang vor der Sicherung von Absatzmärkten eingeräumt worden sei (Sombart 1987: 70). Joachim Radkau wies in seinem Überblickswerk Technik in Deutschland auf die „Dynamik der Sparsamkeit" hin, die in dem deutschen Sparsamkeitsgebot und der Rationalisierungsbewegung ihren Höhepunkt gefunden hätte (Radkau 1989: 107114, 277, 287 f.). Helmut Maier macht deutlich, dass die kriegswirtschaftlich motivierte Substitution von Sparmetallen während der NS-Zeit Bestandteil interdisziplinärer Forschungsvorhaben war (Maier 2007: 1066-1070).

Eine Rohstofforganisation zur Durchsetzung eines blockadefesten, das heißt autarken Staats wurde erst nach Beginn des Ersten Weltkriegs ins Leben gerufen (Burchardt 1968). Die Kriegsrohstoffabteilung im Preußischen Kriegsministerium verfügte anfangs über fünf Mitarbeiter während bei Kriegsende 2.500 Personen dort tätig waren. Sie repräsentierte damit das zentrale Lenkungsorgan der deutschen Kriegswirtschaft (Rathenau 1916, Pleich 1918: 110-151, Wiedenfeld 1936). Diese sowie ähnlichen Zwecken dienende Behörden verwalteten den kriegsbedingten Materialmangel. Darüber hinaus verpflichteten sie andere Institutionen zu vergleichbaren Maßnahmen. Auch die naturwissenschaftlich-technische Forschung 
konzentrierte sich auf Arbeiten, welche die kurze Rohstoffdecke strecken sollten. Neben das Forschungsziel Materialeinsparung traten Untersuchungen zum Wiederverwerten und Ersetzen knapper Stoffe. Wissenschaft und Forschung, so zeigen manche historiographische Befunde, stellten sich in den Dienst einer kriegsbedingten „Ersatzstoffkultur“ (Wengenroth 2002: 59) oder auch „substitute economy " (Lesser 1989: IX, 2, 5, 36). Nun wurden in der Technikgeschichte schon immer Stoffe durch andere, leistungsfähigere, beispielsweise Umwelt und Gesundheit zuträglichere ersetzt, ähnlich wie alte Technologien durch neue abgelöst wurden. In einigen Spezialfällen wurden bereits im 19. Jahrhundert die Begriffe „Ersatz“ (z. B. Grouven 1873), „Ersatzmittel“ (z. B. Frech 1847) oder "Ersatzstoff" (z. B. Hoyer 1887) verwendet. Doch nur wenige dieser Vorschläge wurden im 19. Jahrhundert in nennenswertem Maßstab realisiert. Ein wichtige Ausnahme stellte die Industrie synthetischer Farbstoffe dar (Reinhardt 1997).

Erst die Ausnahmesituation des Ersten Weltkriegs mit einer Importblockade bisher unbekannten Ausmaßes veränderte die Situation grundlegend. Von diesem Zeitpunkt an waren strategische industrielle Basismaterialien für Deutschland auf dem Weltmarkt zu keinem Preis mehr beschaffbar. Nun wurde der Begriff der ,Ersatzstoffe' in Verbindung mit dem Stoffsparen populär. Substitute wurden nun flächendeckend und rasch, an konkreten Stellen und unabhängig von dem dafür bezahlten Preis benötigt. Ersatzstoffforschung ist daher als Gesamtheit all jener naturwissenschaftlichen, technischen und ökonomischen Untersuchungen zu verstehen, die helfen sollten, die politische beziehungsweise kriegsbedingte Knappheit an gewerblichen Stoffen zu lindern. ${ }^{1}$ Der Chemie-Ingenieur Heinrich Mäkelt definierte den Begriff ,Ersatzstoff 1921 folgendermaßen:

[E]in relativer, von den wirtschaftlichen Verhältnissen abhängiger Begriff, insofern als in einem bestimmten Falle zwei Metalle oder überhaupt Stoffe einander vertreten können, je nachdem ob der eine oder der andere billiger und leichter beschaffbar ist, bis zu einer gewissen Grenze auch ohne Rücksicht auf ihre Eignung" (Mäkelt 1921: 191 f.).

In der Literatur kann man lesen, dass eine derartige Ersatzstoffforschung alleine in Deutschland epochenübergreifend Platz gegriffen hätte (Wengenroth 2002). Aber auch auf alliierter Seite gab es während beider Weltkriege, wenn auch in geringerem Maß, staatlich gesteuerte Ersatzstoffforschungen. (Smith 1919: 337-360). Zudem orientierten sich im Jahrzehnt nach dem Ersten Weltkrieg alle westlichen Marktwirtschaften zunehmend am Leitbild des freien Welthandels. Dieser Umorientierung folgte die einschlägige naturwissenschaftlich-technische Forschung, welche die nationalen, kriegswissenschaftlich motivierten Ziele nach und nach verließ. Das gilt auch für die Weimarer Republik, die ab Mitte der 1920er Jahre eine stark exportorientierte Wirtschaftspolitik verfolgte (Schulz 1997: 69-71, Buchheim 2002), deren international ausgerichtete Werkstoffpolitik und Werkstoffforschung auch öffentlichkeitswirksam kommuniziert wurde (Luxbacher 2006). 
Bislang wurden die Voraussetzungen, Bedingungen, Probleme, Lösungsansätze und Erfolge der Ersatzstoffforschung im 20. Jahrhundert noch nicht systematisch untersucht. Auch auf Überblickswerke zum Roh- und Werkstoffmanagement in den beiden behandelten Zeitabschnitten kann nicht zurückgegriffen werden. In einigen Studien wurde zumindest auf Beispiele verwiesen (Petzina 1968: 96-109, Teichert 1984: 181 f., 247, van de Kerkhof 2006).

In den meisten historischen Untersuchungen wird die Ersatzstoffforschung naturwissenschaftlich orientierten Institutionen wie der KaiserWilhelm-Stiftung für kriegstechnische Wissenschaften, den Instituten der Kaiser-Wilhelm-Gesellschaft oder der universitären Forschung (Rasch 1991, Marsch 2000: 326, 426) und damit verbundenen naturwissenschaftlich-technischen Forschungsverbünden zugeordnet (Maier 2007: 419-440, 1066-1070, Luxbacher 2004, 2007, 2010, Flachowsky 2008: 257-259, 322-326).

Der vorliegende Beitrag verfolgt einen neuen Ansatz. Er orientiert sich vorrangig an der Frage nach dem Charakter der deutschen Metall-Ersatzstoffforschung in seiner technischen und betrieblichen Praxis. Aus arbeitsökonomischen Gründen beschränkt er sich vorerst auf gedruckte Quellen. Zunächst ist nach den Zielen und Strategien einer industriellen Werkstoffpolitik in Deutschland in den beiden Autarkieperioden zu fragen. Dann werden die wichtigsten Personen, Institutionen und Ansatzpunkte beleuchtet. Schließlich wird auf der Ebene konkreter Produkte dargestellt, welche Werkstoffe wie eingespart und ersetzt wurden. Es sei vorweggenommen, dass die Forschung an Ersatzstoffen mehr war als das Züchten von Stoffen bestimmter Eigenschaften. Denn die Änderung einer Materialpalette zog tiefgreifende Folgen in den Bereichen Konstruktion, Entwicklung, Erprobung, Gebrauchswert und Nutzung nach sich.

\section{Institutioneller Rahmen im Ersten Weltkrieg}

Die einschneidenden staatlichen Maßnahmen zu Beginn des Ersten Weltkriegs und während der NS-Zeit sind vielfach als „Zwangswirtschaft" oder „gelenkte Wirtschaft" beschrieben worden (Petzina 1968: 153-180, Kahn 2006: 17-25, 417-420, 442-446). Als zentrale Stellen staatlicher Rohstofflenkung fungierte im Ersten Weltkrieg die Kriegsrohstoffabteilung des Kriegsministeriums (KRA). Noch 1914 wurden auch die Ingenieure in das staatliche Roh- und Werkstoffmanagement einbezogen. Als erstes und vielleicht prominentestes Beispiel dafür gilt im Bereich der chemischen Industrie das sogenannte „Salpeterversprechen“2 durch Carl Bosch (Szöllösi-Janze 1998: 283-289).

Die Bereitstellung von Ressourcen für die Erforschung von Stoffeinsparmöglichkeiten und Ersatzstoffen lag prinzipiell nicht im wirtschaftlichen 
Interesse der metallerzeugenden und -verarbeitenden Industrie. Falls sie sich nicht selbst dazu motivierte, musste sie von staatlichen Stellen dazu angehalten werden. Eine Aufzählung aller Verordnungen der KRA füllte vier eng bedruckte Seiten eines Buchs, das nach dem Ersten Weltkrieg vom Mitarbeiter der staatlich kontrollierten Metallfreigabestelle, Arthur Kessner, herausgegeben wurde (Kessner 1921: 25 f.). Die Metallfreigabestelle diente dem Zweck, Gutachten zur Verwendung von Ersatzmetallen sowie entsprechende Freigabeanträge $\mathrm{zu}$ verfassen und „die Industrie zur Verwendung von Ersatzmetallen mehr und mehr zu erziehen" (Kessner 1915: 3). Sie war im Berliner Haus des Vereins Deutscher Ingenieure untergebracht und wurde von dem im Verein Deutscher Ingenieure (VDI) verankerten Maschinenbauprofessor an der Technischen Hochschule Berlin-Charlottenburg, dem Geheimen Regierungsrat Otto Kammerer, geleitet (Rürup 1979: 278, Ludwig/König 1981: 272). Kammerer standen in der Metallfreigabestelle mehrere Chemiker und Ingenieure zur Verfügung (Kessner 1915: 3). Es war also nicht die Industrie selbst, sondern die Metallfreigabestelle, welche die Gründung und Kooperation von Metallberatungs- und Verteilungsstellen in den einzelnen Industriezweigen vorantrieb. ${ }^{3}$ So bedauerte der mit Ersatzstoffarbeiten betraute Maschineningenieur Georg von Hanffstengel, dass der Ersatz kupferner Armaturen durch eiserne, „nur sehr ungern und zögernd“ in Angriff genommen worden sei. ${ }^{4}$ Eine ganze Reihe von Industrien wehrte sich offen gegen die staatlichen Zwangsmaßnahmen und die Verwendung daraus resultierender, weitgehend unsicherer Substitute (Leiser 1921: 170). Das wesentliche Motiv der Industrie beim Werkstoffsparen und -ersetzen war während des Ersten Weltkriegs nicht ein wirtschaftlicher Anreiz, sondern Nationalbewusstsein, staatlicher Druck und Strafdrohungen bei „Rohstoffvergehen“ (Maier 2002: 363, Kessner 1915: 2).

Immer mehr Metalle mutierten im Kriegsverlauf zu „Sparstoffen“ und damit zu knappen Materialien (Maier 2002: 363-371, Dettmar 1916), im Gegensatz zu „Heimstoffen“. Die Metallberatungs- und Verteilungsstellen arbeiteten ab 1915 Dringlichkeitspläne und Rangfolgen dieser knappen Metalle aus. Diese Listen änderten sich während des Kriegsverlaufs immer wieder und wurden ständig länger. Im April 1918 lautete die Liste in absteigender Reihenfolge der Knappheit: Industriediamanten, Platin, Wolfram, Molybdän, Vanadium, Silber, Nickel, Zinn, Quecksilber, Kupfer, Chrom, Qualitätsgraphit, Aluminium, Antimon, Blei und Zink. Es wurde als Erfolg verbucht, wenn man einen Stoff mit niedriger Nummer durch einen Stoff mit höherer Nummer ersetzen konnte. Als noch größerer Erfolg galt, wenn es gelang ihn durch Heimstoffe zu ersetzen: Stahl und Eisen, amorpher Graphit, Hartholz, Hartpapier, Pappe, Glas und Steinzeug. Sparmetalle wurden ausschließlich auf der Basis von amtlich genehmigten Beleg- oder Freigabescheinen ausgegeben. Dafür war der Nachweis der Unersetzlichkeit der Materialien zu erbringen beziehungsweise der Nachweis über eine Kriegsnotwendigkeit (Kessner 1921: 29-31). Die Appelle an die Industrie, Freigabeanträge für Sparstoffe erst nach sehr gründlicher Prüfung von dafür 
möglicherweise geeigneten Heimstoffen zu stellen, wurden immer dringlicher. Mitunter empfahlen die staatlichen Beauftragten der Industrie, sogar dann Ersatzstoffe zu nutzen, wenn eine geringere Lebensdauer und Wirtschaftlichkeit zu erwarten war. Im Zweifel sollte man eine Vorprüfung durch Spezialisten des Industrieverbandes vornehmen lassen (ebd.: 32).

Georg von Hanffstengel berichtete 1916 vor dem VDI Karlsruhe über die vordringlichsten Probleme. An erster Stelle stünde Kupfer, vor allem in seinen Legierungen mit Zink als Messing und Rotguss sowie mit Zinn als Bronze. Diese wurden vor allem für Maschinenlager und -getriebe benötigt. Hanffstengels Referat lässt sich entnehmen, dass den Anregungen der Beratungsstellen vor allem eine intensive Gebrauchswertforschung zugrunde lag. Ziel war die Verwendung des unter kriegswirtschaftlichen Bedingungen jeweils am leichtesten greifbaren Stoffes, der gerade noch den jeweiligen technischen Anforderungen entsprach. So regte Hanffstengel an, das Sparmetall Messing zu verwenden, wenn es möglich sei, damit noch schwieriger zu beschaffende Metalle, wie etwa reines Kupfer, zu ersetzen. Dies sei etwa bei bestimmten chemischen Einflüssen möglich. Hanffstengel riet dazu, das Vernickeln durch das Verkobalten zu ersetzen, obwohl dies „erheblich teurer“ sei (Hanffstengel 1916: ohne Seitenangabe). Sein Vortrag verdeutlicht, dass bei der Frage des Werkstoffersatzes weniger der ökonomische Vorteil als vielmehr die kriegswirtschaftliche Notwendigkeit im Vordergrund stand.

\section{Institutioneller Rahmen in der NS-Zeit}

Die institutionellen Organisationsmuster aus dem Ersten Weltkriegs kehrten während der Zeit des Nationalsozialismus wieder. Auf juristisch-wirtschaftspolitischer Seite war es anstelle der KRA nun die staatliche VierjahresplanAgentur „Reichsamt für Wirtschaftsausbau“. Hinzu kamen zahlreiche Reichsstellen wie die Reichsstelle für Metalle, die etwa für den Maschinenbau und die Elektroindustrie detailliert und verbindlich festlegte, welche Metalle nur noch bedingt, für welche Einsatzzwecke, und welche gar nicht mehr verwendet werden durften (Eberhardt 1938, Arbeitsgemeinschaft zur Förderung der Elektrowirtschaft 1944). Abermals erteilte der VDI Ersatzstofflektionen. Der Zweck dieser Veranstaltungen lag darin, so Hans Herttrich, Ingenieur bei der Reichsstelle für Metalle, „der Welt den Erfolg der Lösung unserer Rohstoffprobleme als Beispiel unbeirrbarer Ingenieurarbeit nachzuweisen." (vgl. Maier 2007: 921). Dabei kooperierte der VDI, wie schon während des Ersten Weltkriegs, mit einer Reihe staatlicher Organisationen wie der Reichsstelle für Metalle, dem Reichsminister für Bewaffnung und Munition sowie dessen Sparkommissaren, dem Bevollmächtigten für die Maschinenproduktion und den Wehrkreisbeauftragten (Herttrich 1940: 1-4). 
Auch hier stand wieder ein enges Geflecht staatlicher und privater Institutionen mit Ingenieuren im Zentrum staatlicher Werkstoffpolitik. Bestes Beispiel dafür ist Georg Hanffstengel, der während der Weimarer Republik mit ganz anderen Aufgaben betraut war, nun aber wieder in der Ersatzstoffforschung tätig wurde. ${ }^{5}$ So gab er ab 1936 die Schriftenreihe Ingenieurfortbildung heraus, in der 1937 ein Band von Heinrich Bürgel über Deutsche Austauschwerkstoffe erschien (Bürgel 1937). Gemeinsam fassten sie die im Sinne der nationalsozialistischen Autarkiepolitik zu ergreifenden Maßnahmen folgendermaßen zusammen: sparsamste Bewirtschaftung der vorhandenen deutschen Rohstoffvorräte, Verbesserung der Gewinnungsverfahren durch Vermeidung von Rohstoffverlusten, Einführung neuartiger, Abfall sparender Produktionsverfahren und stärkere Nutzung von ,Heimstoffen' beziehungsweise ,Reichsstoffen'. Bei letztgenannter Maßnahme sollten die bisher benutzten Werkstoffe unter weitestgehender Vermeidung und Einsparung devisenpflichtiger Auslandsstoffe planmäßig veredelt und hoch gezüchtet werden. Gleichzeitig warnten sie jedoch vor einer „Überspannung“ der Sparmaßahmen, da dann eine "Güteminderung der deutschen Maschinen“ drohe und dies „zu einer Schädigung des Ansehens der deutschen Technik führen" könne (Hanffstengel/Bürgel 1937: Vorwort).

Der wichtigste Weg, so das Fazit der beiden, bestünde jedoch darin, „,neue deutsche Werkstoffe“ zu schaffen. Diese müssten „ebenso gut, wenn nicht besser sein, als die bisher benutzten, so daß bei ihrer Verwendung keine Güteminderung, sondern eher eine Gütesteigerung eintritt" (ebd.). Damit lagen sie völlig auf dem Kurs der offiziellen Propaganda zum Vierjahresplan, welche die angeblich gleichwertige, wenn nicht überlegene Qualität „deutscher Werkstoffe" verkündete (Adler 1939).

Auch Herttrichs Hauptsorge galt, aufbauend auf den Erfahrungen des Ersten Weltkriegs, der hohen Produktqualität. Er mahnte, dass bei der Umstellung auf „Austauschstoffe“ „stets die Gütefrage entscheidend sein" müsse (Herttrich 1940: 1). Im nächsten Satz deutete er das ganze Dilemma der Ersatzstoffforschung an: „Wo eine Güteminderung nicht zugelassen werden kann, soll die Umstellaufgabe neu geprüft werden." (Ebd.). Um den aus dem Ersten Weltkrieg negativ konnotierten Begriff ,Ersatzstoffe' zu umgehen (Maier 2002: 359), forcierte die Vierjahresplanbehörde in Zusammenarbeit mit dem Deutschen Werberat den Begriff des ,Austauschstoffes'. An der Strategie änderte dies freilich nichts (Luxbacher 2006: 13).

\section{Ersatzstoffforschung als Forschung der betrieblichen Praxis}

Es bestanden also zahlreiche politische und ökonomische Parallelen bei der Beschaffung industrieller Roh- und Werkstoffe zwischen den beiden 
Autarkiephasen. Davon ausgehend wird im Folgenden nach den wesentlichen Intentionen, Strategien und Orten der Ersatzstoffforschung beider Perioden gefragt.

Den genannten Forschungen lag sowohl naturwissenschaftliches und technikwissenschaftliches als auch praktisches beziehungsweise Erfahrungswissen zugrunde. Das Kernproblem aber hatte politisch-ökonomischen Charakter, nämlich die Einsparung bestimmter Materialien bei der Herstellung technischer Produkte. Diese bestanden aus den verschiedensten, über Jahrzehnte erprobten Werkstoffen. Jeder dieser Stoffe verfügte über bestimmte Eigenschaften. Von diesen wussten die Konstrukteure und Maschinenbauingenieure aus Erfahrung, dass ihr Einsatz an bestimmten Stellen üblich vorteilhaft oder unbedingt notwendig war. Worüber sie jedoch kaum Detailwissen besaßen, war das Verhalten eines Bauteils innerhalb der jeweiligen Konstruktion, wenn dieses aus einem anderen Werkstoff gefertigt worden war. Zwar hatten die Metallographie und Metallphysik sowie die Materialprüfung und vor allem die technische Mechanik in der Zwischenkriegszeit große Fortschritte gemacht. Doch zur Erforschung von Bauteilen aus ungewöhnlichen Materialien beim Betrieb von Apparaten, Geräten und Maschinen hatte es während der exportorientierten Weimarer Republik wenig Veranlassung gegeben. Anders in den beiden nach Autarkie strebenden Epochen. Betrachtet man das Geschehen in deren Produktionsstätten, zeigt sich, dass dabei das praktische und das Erfahrungswissen von essentieller Bedeutung waren.

\section{Nicht systemgebundene Produkte}

Am einfachsten waren Sparmetalle bei Gegenständen des alltäglichen Gebrauchs zu ersetzen, die nicht den technischen Normen einer größeren Konstruktion oder eines Netzwerks folgen mussten. Essbesteck aus Messing, Neusilber oder sonstigen Nickel-Kupfer-Legierungen konnte durch Eisenblech mit einem Überzug aus Messing oder Zinn substituiert werden. Ein derartiges Vorgehen bei Konsumgütern benötigte vergleichsweise geringe werkstoff- oder konstruktionstechnische Expertise (Leiser 1921: 168-170). Auch bei Hand- und einigen Maschinenwerkzeugen waren Erfolge bei der Einsparung von Mangelmetallen zu verzeichnen. So zeigten zum Beispiel Untersuchungen, dass beim Schnellstahl ohne größere Qualitätseinbußen der bis dahin übliche Wolframgehalt von 18 Prozent auf elf Prozent herabgesetzt werden konnte. Dafür musste in vielen Fällen ein Qualitätsausgleich durch Maßnahmen wie zusätzliche Härteverfahren oder Einhalten genauerer Temperaturvorgaben Schmelz- und Kühlprozessen geschaffen werden (Verein Deutscher Ingenieure 1940: 37-39). 


\section{Systemkomponenten}

Wesentlich schwieriger gestaltete sich das Werkstoffsparen und -ersetzen bei technischen Systemkomponenten. Bei Anzweigstutzen von Hochdruckleitungen wurden nur die besonders belasteten Bauteile in Rotguss belassen, der Rest in Pressstahl ausgeführt (vgl. Abb. 1). Doch selbst bei diesen relativ einfachen Modulen waren deshalb konstruktive Änderungen nötig.

Ähnlich in der Elektrotechnik. Zwar war Aluminium prinzipiell an Stelle von Kupfer als Leitungsmaterial einsetzbar. Doch mussten dazu zahlreiche fertigungstechnische und konstruktive Systemkomponenten umgestellt werden. So gab es Probleme bei der Verwendung von Aluminiumdrähten, die Kupfer ersetzen sollten, da bei den Montageverbindungsstellen mit anderen Materialien verstärkt Korrosion auftrat (Schwarz 1993). Ein defektes Bauteil konnte den Stillstand eines ganzen Systems verursachen. Nicht zuletzt aus diesem Grund wurde zur Zeit des Ersten Weltkriegs der Begriff ,Ersatzstoffe' zunehmend negativ konnotiert (Maier 2007: 290, Luxbacher 2006: 9). Hinzu kam, dass das Angebot vieler als angeblich tauglich angebotenen Ersatzstoffe im Laufe der Kriegsjahre sogar für „Sonderfachleute“ unübersehbar wurde: „Wenn auch in Fachzeitschriften viel über Ersatzstoffe aller Art berichtet wurde, so fehlten doch meist die den Verbrauch in erster Linie angehenden Angaben über ihre Bewährung in der Praxis." (Kessner 1921: III).

Während des Ersten Weltkriegs nahm Otto Kammerer eigene metallographische Forschungen zu sparstofffreien Maschinen- und Eisenbahnlagern auf (ebd.: 107-110). Parallel dazu arbeitete der Versuchsstand im metal-

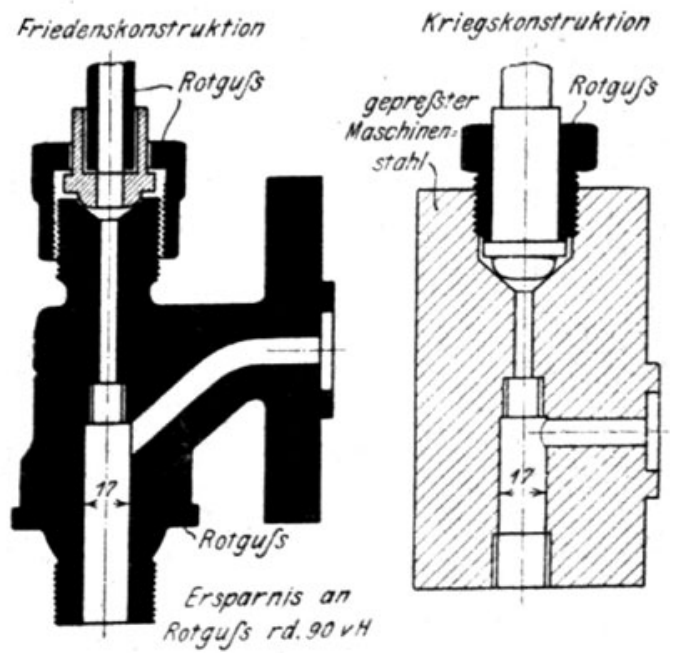

Abb. 1 Abzweigstutzen für Hochdruckleitungen in Friedens- und Kriegsbauart. Rotguss (schwarz) wird nur noch an kritischer Stelle verwendet, ansonsten gebohrter Pressstahl (schraffiert). (Kessner 1921: 163, Abb. 98) 
lographischen Laboratorium des Eisenhüttenmännischen Instituts der Technischen Hochschule Berlin-Charlottenburg unter dem Metallographen Heinrich Hanemann und mit Georg Hanffstengel an der Minimierung von Lagerreibung. Während Kammerer jedoch nach verallgemeinerbaren Phänomenen suchte, arbeiteten Hanemann und Hanffstengel praxisnäher. Sie fokussierten nicht nur auf den Werkstoff, sondern auch auf die Wechselwirkungen mit der jeweiligen konstruktiven Eigenart (Arten und Dauer der Belastung), deren Einsatzweise und Einwirkungen des jeweiligen Betriebsortes (wie Temperatur und Luftfeuchtigkeit). Sie nahmen sich jede einzelne Lagerkonstruktion auf ihre Brauchbarkeit für einen bestimmten Zweck hin vor (ebd.: 110-115). Betrug der Anteil des Sparmetalls Zinn in einschlägigen Lagermetallen vor 1914 zwischen 80 und 86,1 Prozent gegenüber dem Rest Heimstoffe, so drehte sich diese Prozentverhältnis während des Kriegs häufig zu Gunsten der Heimstoffe um (ebd.: 125). Derartigen Arbeiten entsprang etwa eine Legierung aus Blei mit fünf Prozent Zinn, 15 Prozent Antimon und circa einem Prozent Kupfer. Ein solches „Einheitsmetall“ sollte für Achslager von Güterzugwaggons verwendet werden. Aber erst umfangreiche praktische Erfahrungen zeigten, dass diese Legierung nur für bestimmte Spezialanwendungen tauglich war (ebd.).

Im Bereich der Elektrotechnik wurden im Dezember 1914 erste Kriegsverordnungen und Normalien durch den Verband deutscher Elektrotechniker (VDE) erlassen. Der ,Heimstoff' Zink spielte dabei eine wesentliche Rolle als Ersatzstoff. Unter Federführung des Generalsekretärs des VDE, Georg Dettmar, wurden "Ausnahmebestimmungen während des Krieges“ in fünf Auflagen zwischen 1915 und 1918 zusammengestellt (Wronkow 1921: 42-54). Dabei spielten physikalische und chemische Analysen für den Praktiker Dettmar kaum eine Rolle. Der maßgebliche Partner der Betriebsingenieure waren die Technikwissenschaften und in noch größerem Maße die Industrie (Kessner 1915: 546 f.). Betriebsingenieure und Konstrukteure hatten andere Sorgen, als die analytischen Ergebnisse zu binären Legierungen oder Drei- und Vierstoffsystemen abzuwarten. Sie beschäftigte eine Vielzahl von Fragen zu denen unter anderem die Härte, die Plastizität oder rationellere Produktionsmethoden gehörten (Kessner 1921: 104 f.).

Zu den praktischen Problemen zählten auch Neukonstruktionen von Systemkomponenten wie elektrische Isolatoren und Gestänge aus Heimstoffen. Dabei stellte sich beispielsweise heraus, dass deren Einsatz „wesentlich höhere Kosten" verursachte als bei Systemkomponenten, die auf Kupferleitungen ausgelegt waren (Wronkow 1921: 54). „Damit müssen wir uns aber in der Kriegszeit abfinden und ausdrücklich betonen, dass Wirtschaftlichkeit und Wirkungsgrad bei allen Ersatzstoffen etwas in den Hintergrund treten müssen." (Ebd.)

Allenfalls interessierten sich Ingenieure für bestimmte, die Leistungsfähigkeit von technischen Systemen beeinträchtigende naturwissenschaftliche Phänomene: so etwa für den noch nicht verstandenen Skin-Effekt in 
Kupferleitern, der bei Eisenleitern noch verschärfter auftrat. Nur durch praktische Versuche und Erprobungen konnte man die Verluste in Grenzen halten (Wronkow 1921: 42-54).

Obwohl nach dem Ersten Weltkrieg in der Metallkunde Fortschritte erzielt werden konnten, zeichneten sich auch in der NS-Autarkiepolitik ab 1933 strukturell vergleichbare Schwierigkeiten ab. Um Kupfer einzusparen, setzte man Cupal, einen kupferplattierten Aluminiumdraht, ein. Solche Drähte vereinigten in sich den Vorteil des geringeren Gewichtes mit denen einer äußeren Kupferhülle, so dass der Skin-Effekt sich nicht mehr so negativ bemerkbar machte. Doch diese Technologie hatte ihren Preis. Die Aluminiumleitungen gleicher Leistung mussten wesentlich dicker ausgeführt werden als die kupfernen. Im Falle der stark miteinander vernetzten Systemkomponenten hatte dies massive Auswirkungen auf das gesamte elektrotechnische System „, so dass bereits bei der Konstruktion entsprechend mehr Platz vorgesehen werden muss." (Verein Deutscher Ingenieure 1940: 40). Doch gerade Neukonstruktionen mussten unter Kriegsbedingungen vermieden werden, da sie zu viele materielle und geistige Ressourcen banden. Ähnliche werkstoffgerechte Um- und Neukonstruktionen wären auch in vielen anderen Bereichen des Maschinenbaus nötig gewesen (Kessner 1940: 45-48). Daher waren Betriebserfahrung und Improvisationsfähigkeit im Allgemeinen wichtiger als im Labor und auf dem Prüfstand gewonnenes Wissen.

\section{Die ungeahnte Komplexität der Ersatzstoffforschung}

Die Komplexität des Verhältnisses zwischen Werkstofftechnik, Konstruktion und praktischem Betrieb bereitete der Werkstoffumstellung massive, teilweise vorher nicht bedachte Probleme. Jeder Werkstoff war ganz spezifischen Beanspruchungen ausgesetzt. Zusätzlich konnten diese Beanspruchungen je nach Gestalt der Bauteile und deren Zusammenwirken modifiziert werden. Wie Maier schreibt (2002: 378), scheint „ jedes Umstellvorhaben seine eigene Biographie gehabt zu haben“. Nachdem August Wöhler bereits Mitte des 19. Jahrhunderts dynamische Bauteilbelastungen gemessen hatte, wurde in der Technischen Mechanik um 1900 damit begonnen, derartige Fragen zu messen und zu mathematisieren. Doch erst in der Zwischenkriegszeit begannen Maschinenwissenschaftler wie der an der Technischen Hochschule Darmstadt wirkende August Thum damit, diese Zusammenhänge systematisch zu analysieren, allerdings zunächst für die traditionell verwendeten Werkstoffe (Thum 1944). Während der Zeit des Nationalsozialismus konzentrierte sich dann auch Thum auf die staatlich vorgeschriebenen Materialien. Doch die „Klärung der tatsächlich in den Maschinen auftretenden Betriebsbeanspruchungen und der tatsächlich im Betrieb zur Geltung kommenden Werkstoffeigenschaften“ entpuppte sich als gewaltiges Grundlagenforschungsprojekt weniger der Natur- als 
der Technikwissenschaften und letztlich der Werkstatttechnik. (Uhde 1941: 161). Zwar bemühte man sich, einschlägige Messungen und Berechnungen zu generalisieren. Doch die Kombination von vielfältigen Belastungsarten mit einer hohen Anzahl von Mutationen bei Konstruktionen machte Vorhersagen schwierig bis unmöglich.

Die Versuche der Metallberatungs- und Verteilungsstelle zeigten, dass man sich dem Werkstoffproblem letztlich nur auf dem Weg des maschinentechnischen Experiments und Ausprobierens nähern konnte. Die technische Materie war zu vielschichtig und komplex, als dass auf allgemeinen Prinzipien beruhende Lösungen gefunden werden konnten. Die jeweiligen Beanspruchungen und Betriebsbedingungen waren häufig nur fallbezogen fassbar. Das lässt sich auch daran erkennen, dass die „Drucksachen“ und die „Berichte“ der Metallberatungs- und Verteilungsstellen des Vereins Deutscher Maschinenund Anlagenbau (VDMA) gegen Kriegsende eine stattliche Reihe von Bänden mit zahlreichen Einzelbeispielen umfassten. ${ }^{6}$ Ersatzstoffforschung bedeutete eher ein systematisches Ausprobieren und Beobachten von Spezialfällen in der Werkstatt als die Entwicklung bestimmter Legierungen oder die Manipulation von Kristallgittern im Labor. Die Kenntnis der Bauteilform und die auf sie wirkenden Lastkollektive sowie thermische und chemische Einflüsse waren von größerer Bedeutung als die Präzisierung von Mikrostrukturen. Unter den in Frage kommenden Materialien galt es schließlich jene auszuwählen, die den Ansprüchen voraussichtlich gerade noch genügten. Eine weitere Möglichkeit bestand darin, die Konstruktion so zu ändern, dass möglichst wenige Sparstoffmengen verbaut werden mussten. Ersatzstoffforschung erhielt dadurch unfreiwillig experimentellen Charakter mit dem „Zweck, für bestimmte Anwendungsgebiete best geeignete Werkstoffe zu finden“. 7 Bei diesem Experiment stellten Erkenntnisse der Materialprüfung, der Metallographie und der Metallphysik nur einige von vielen Informationsquellen der Ingenieure dar.

Georg von Hanffstengel resümierte:

[Die] Einflüsse sind unendlich mannigfaltiger Art, und das Verhalten der Metalle weist unter scheinbar ähnlichen Verhältnissen so weitgehende Verschiedenheiten auf, daß es wohl verständlich ist, wenn man im Maschinen- und Apparatebau zunächst den Wunsch hatte, nach Möglichkeit bei den alten Baustoffen zu bleiben. ${ }^{8}$

Unterschiedliche Konzentrationsgrade, Temperaturen, Flüssigkeitsbewegungen, Wasserqualitäten und dergleichen „führen oft zu Erscheinungen, die einander völlig zu widersprechen scheinen und ein Gefühl der Unsicherheit gegenüber diesen Stoffen aufkommen lassen." Viele Ersatzstoffe, so Hanffstengel, seien etwa wegen des darin enthaltenen Zinks nicht so widerstandsfähig „und es ist daher nicht richtig, sie zu verwenden, wenn bestimmt mit einer ganz erheblich verkürzten Lebensdauer gerechnet werden muß, so daß mehrere Stücke aus Messing statt eines Stückes aus Bronze aufgebraucht würden."9

Ähnliches zeigte sich beim Austauschen von Walzwerkslagern. Zinn und Kupfer sollten, so der mit Ersatzstoffen befasste Betriebsingenieur M. Leiser, 
für „unmittelbare Kriegslieferungen“ bereitgehalten werden, weshalb die aus hochwertiger Bronze bestehenden Lagerschalen durch weniger wertvolle Stoffe zu ersetzen waren (Leiser 1921: 179). Umfangreiche Versuche wurden mit Zink- und Bleilegierungen, und sogar unterschiedlichen Holzsorten unternommen. In allen Fällen waren leidlich brauchbare Ergebnisse nur durch jahrelanges Sammeln praktischer Erfahrungen bei der Herstellung und im Betrieb zu erreichen. Die grundsätzliche Problematik bestand darin, je nach Lagerund Maschinenart das richtige Material zu finden und dieses richtig zu dimensionieren und $\mathrm{zu}$ formen, $\mathrm{um}$ es in die restliche Neu- oder Umkonstruktion einzupassen und eine gewisse Mindestbelastbarkeit und -haltbarkeit zu erreichen. Werkstoffgerechte Konstruktion und (bei Umbauten) deren Improvisation war gefragt. Manche Lager wurden gleichmäßig belastet, andere waren beispielsweise schweren Stößen ausgesetzt. Leichte, mittlere und schwere Walzstraßen erforderten jeweils ganz andere Lösungen. Hinzu kamen unter anderem Schwierigkeiten mit der Maßhaltigkeit, den Einpassungen, der Schmierung, der Rissgefahr oder der chemischen Behandlung. Bei manchen Lagerarten war das Problem einfach nicht lösbar. So erkannte man nach all den Mühen, dass man bei Kaltwalzwerken um den Einbau von kostbaren Rotgusslagern einfach nicht umhin kam. Auch bei den Hauptdrucklagern der schweren Walzstraßen war der Ersatz von Bronze völlig fallabhängig und „nur in beschränktem Umfange möglich. Ganze Lager aus Zinklegierungen haben bei schweren Straßen nicht gehalten, sie sind zu spröde und zerspringen unter den heftigen Stößen in viele Stücke" (ebd.). Ganz offensichtlich lagen die Möglichkeiten, die sich dem Ersatz hier boten, bei jedem einzelnen Unternehmen und Walzwerk anders. Dementsprechend negativ fiel ein Rückblick über das im Ersten Weltkrieg Erreichte auf dem gesamten Feld der Antriebs- und Transmissionstechnik aus:

Besonders schlimm ist es für die Werke, daß jetzt alles zusammenkommt: geringwertige Metalle, schlechte Öle und Treibriemen von geringer Güte. Die Schwierigkeiten addieren sich nicht, sie multiplizieren sich. Daher mehren sich die Störungen im Betriebe. (Kammerer 1917: 10)

Während der Zeit des Nationalsozialismus untersuchte der Dresdener Maschinenwissenschaftler Enno Heidebroek vor allem Lagerfragen. Seine wesentliche Erkenntnis bestand ebenfalls darin, dass es weniger um den Einsatz und das Verhalten verschiedener Werkstoffe als vielmehr um grundlegende Fragen wie Reibung und Schmierung ging. Heidebroek hob gegenüber den interessierten Mitgliedern des VDI hervor, dass der ideale, am wenigsten verschleißende Zustand eines Lagers die Schwimmreibung darstelle, also die volle Flüssigkeitsreibung (Heidebroeck 1940: 8-11). Sei dies erreicht, wären alle anderen Kriterien, auch die Materialfrage, so Heidebroek, sekundär. In der Betriebsrealität würde dieser Idealzustand häufig nicht erreicht. Lagerschäden träten vor allem durch die einfache Tatsache „der zu geringen Steifigkeit der Welle“ auf (ebd.: 8). Nur bei „genügender Formsteifigkeit und nicht zu engem 
Lagerspiel" könnte an Austauschwerkstoffe wie Bleilagermetalle, Leichtmetalle und Kunstharz-Pressstoffe gedacht werden (ebd.). Dadurch könne „die Wirkung der hochzinnhaltigen Legierungen annähernd erzielt werden, jedoch ist je nach den Betriebsverhältnissen der eine oder andere Werkstoff vorzuziehen" (ebd.: 10). Dafür sei jedoch ausreichende und je nach Reibungsverhältnissen angepasste Schmierung erforderlich: „Der Einfluss des Schmierstoffs wird in Zukunft von einer wesentlichen höheren Größenordnung sein als die vielfach geringen Unterschiede der einzelnen Legierungen." (ebd.: 11).

\section{Der hohe Preis der Austauscherfolge}

In beiden Autarkiephasen wurden Austauscherfolge erreicht. Doch wie gezeigt, hatten diese ihren mehr oder minder hohen Preis. Eines von vielen typischen Beispielen soll das illustrieren. Unmittelbar nach Kriegsbeginn, im Oktober 1939, erhielt die Schwerindustrie von staatlicher Stelle den Auftrag, Chrom-Molybdän-Stähle durch Chrom-Mangan-Stähle auszutauschen. Molybdän wurde vor allem aus den USA eingeführt. Aus derartigen hochwertigen Baustählen wurden beispielsweise Getriebeteile wie Zahnräder hergestellt. Metallurgische Mikroanalysen im Labor waren nebensächlich. Was zählte war der rasche praktische Erfolg in der Werkstatt. Wie ließen sich die als Ersatz in Frage kommenden mit Mangan und Chrom legierten Baustähle EC-80 und EC-100 als Ersatzstähle bearbeiten? Welcher Wärmebehandlung waren sie zu unterziehen, damit sie in etwa dieselben Eigenschaften wie die Chrom-Molybdän-Stähle aufwiesen? Und welche Festigkeitseigenschaften zeigten sie dann tatsächlich im praktischen Betrieb? Die für diese Zwecke mit gering variierenden, jedoch leichter beschaffbaren Legierungen hergestellten Stahlsorten wurden für die Herstellung mehrerer unterschiedlicher Getriebesorten zunächst abgedreht, gebohrt, geräumt und geschliffen. Die dabei auftretenden Ähnlichkeiten und Unterschiede wurden vermerkt. Anschließend wurden die Zahnräder wärmebehandelt, wobei man feststellte, dass nur "sehr schwach aufkohlendes Einsatzpulver“ verwendet werden konnte, was die Einsatzzeiten erhöhte und damit gleichzeitig die Produktivität deutlich minderte. Der darauf folgende Härtungsprozess war zwar vergleichbar mit dem der bisher eingesetzten Stähle, doch musste mit der Thermalhärtung die schwierigste und kostspieligste Härtungsmethode angewendet werden. Ein derartiges Wärmebehandlungsverfahren wäre bei den herkömmlichen Legierungsstählen nicht erforderlich gewesen. Bei Berücksichtigung all dieser Besonderheiten kam man auf einigermaßen mit den ursprünglichen Werkstoffen vergleichbare Festigkeitswerte (ohne diese jedoch zu übertreffen). Sie wurden in der Schlagbiegeprobemaschine sowie an 
ihrem Endbestimmungsort, sprich: Lkw-Getrieben, erprobt. Parallel laufende Praxistests mit Chrom-Molybdän-Nickel-Stählen, die durch Chrom-Molybdän-Stähle ersetzt werden sollten und für andere Verwendungsfelder gedacht waren, verliefen dagegen ungünstig. Ihre Schlagzähigkeit war zu gering (von Soden/Ulrich 1940: 26-29).

\section{Neukonstruktion und Neudimensionierung}

Auch andere Getriebeteile wurden untersucht. So wurden die Sparstoffe Nickel und Molybdän als Legierungsmetalle für die Stähle von Stirn- und Kegelrädern genutzt, Kupfer, Zinn und Nickel für die Bronzekränze von Schneckenrädern. Hier gab es einen Erfolg zu verzeichnen: Es stellte sich in praktischen Versuchen heraus, dass zumindest im Großgetriebebau, bei unlegierten oder Silizium-Mangan-Stählen ausreichende Walzenfestigkeit und befriedigendes Laufverhalten zu beobachten war. Bei einigen Konstruktionen schien sogar der unter dem Gesichtspunkt der Autarkie besonders vorteilhafte Einsatz von Kunstharz-Pressstoffen machbar. Schwierigkeiten bereiteten die Schneckenräder, wie sie etwa im Pkw-Bau eingesetzt wurden. Hier vereitelte das starke Gleiten längs der Zahnflanke den Übergang zu einem Ersatzmaterial. Diese besondere Laufeigenschaft wurde durch Bronze aufgefangen, die jedoch die Sparstoffe Kupfer und Blei enthielt. Die Ingenieure des VDI testeten einige Leichtmetall-Alternativen sowohl am Maschinen-Prüfstand als auch im praktischen Fahrbetrieb. Als prinzipiell brauchbar erwiesen sich Legierungen aus Aluminium-Silizium, Aluminium-Magnesium-Mangan und AluminiumKupfer-Magnesium. Allerdings zeigten sie wenig Verschleißfestigkeit, zu geringe Schwellfestigkeit und zu hohe Bruchdehnung. Um diese Materialien dennoch einsetzen zu können, mussten die Ingenieure die gesamte Schneckenrad-Konstruktion neu dimensionieren. Die Kranzbreite musste fast verdoppelt werden. Das bedeutete zwar, dass ein derartiges Schneckenrad ähnlich zuverlässig arbeitete wie eines aus herkömmlichem Material, aufgrund der unterschiedlichen Dimensionen aber nicht als Ersatzteil taugte. Erst im Falle einer kompletten Neukonstruktion und damit Vergrößerung der gesamten Anlage konnte dieses Maschinenelement überhaupt eingesetzt werden. Vergleichbares traf auch für alle anderen durchprobierten Werkstoffe $\mathrm{zu}$, so beispielsweise für die Zink-Aluminium-Kupfer-Legierung des schlesischen Zinkherstellers Giesches Erben, aber auch für Gusseisen und Stahl. Allerdings erreichte hierbei nur eine einzige Variante den geringen Abrieb und die Lebensdauer der Bronze-Legierung. Die Lebensdauer erreichte im Falle von Magnesium-Legierungen nicht einmal die Hälfte, und im Falle der diesbezüglich günstigsten Variante mit Aluminium nur etwa einen 20 Prozent geringeren Wert. (Altmann 1940: 29-32). Diese Kennziffern waren obendrein 
noch sehr stark vom jeweiligen Einsatzzweck abhängig, so dass die Autoren bedauerten, dass „es an einer scharfen Abgrenzung der Verwendungsbereiche der neuen Werkstoffe fehlt". Deshalb seien „entsprechende Forschungsarbeiten unerlässlich“ (Altmann 1940: 32). Einmal mehr wird deutlich, dass der Ort der Ersatzstoffforschung in der Werkstatt und weniger im Labor zu finden war. Erst dort zeigte sich, wozu ein Ersatzstoff wirklich taugte.

\section{Der Kampf um den Wirkungsgrad}

Entgegen anderslautender regierungsoffizieller Propaganda in beiden Autarkiephasen mussten bei vielen Produkten immer wieder Qualitätseinbußen hingenommen werden. So formulierte der Chemieingenieur Heinrich Mäkelt 1921:

Es lässt sich also auch in dieser Hinsicht ein Metallersatz nicht ohne weiteres empfehlen oder verwerfen, vielmehr muß in der Folge im einzelnen Falle geprüft und untersucht werden, ob die Aufwendungen für den Ersatz technischen und wirtschaftlichen Forderungen entsprechen oder nicht. Im Kriege hat hierauf durchaus nicht immer Rücksicht genommen werden können. Es wird deshalb auch von solchem Ersatz die Rede sein müssen, der den Ansprüchen in keiner Weise genügt." (Mäkelt 1921: 191 f.).

Bereits während des Ersten Weltkriegs gründete der Verein deutscher Eisenhüttenleute eine eigene Sparmetallkommission für Hochofenarmaturen. Diese Kommission diente als Muster für weitere ähnliche Kommissionen in benachbarten Bereichen (Leiser 1921: 175). Mit akkurater Systematik gingen diese Kommissionen daran, ausbaufähige und austauschbare Teile an bestehenden Anlagen zu lokalisieren. Der damit verbundene Aufwand an Forschung, Erprobung, Herstellungs-, Transport- und (De)montagearbeiten wäre in Friedenszeiten als nicht tragbar angesehen worden. Funktionsfähige kupferne Hochofenwindformen wurden durch eiserne ersetzt, um das Kupfer verwerten zu können. Deren Betrieb benötigte allerdings die doppelte Menge Kühlwasser, weshalb $\mathrm{Zu}$ - und Ableitungen neu dimensioniert und zusätzliche Leitungen installiert werden mussten. Zudem war deren Lebensdauer geringer. Dennoch wurden während des Kriegs etwa zwei Drittel aller kupfernen und sogar bronzenen Windformen in deutschen Thomaswerken ausgebaut und ersetzt (Leiser 1921: $176 \mathrm{f}$.).

Bald nach Beginn des Ersten Weltkriegs wurde damit begonnen, die Kupferwicklungen elektrischer Maschinen durch Zinkwicklungen zu substituieren. Da Zink einen deutlich schlechteren Leiterwerkstoff darstellt, verringerte sich der Wirkungsgrad der Maschinen beträchtlich. Als ab 1916 größere Mengen von im Inland gewonnenen Aluminium bereitstanden, begannen die Elektroingenieure auf Empfehlung des VDE die Zinkwicklungen durch Aluminiumwicklungen auszuwechseln, die wiederum einen etwas besseren Wirkungsgrad als Zink aufwiesen. 1916 erschienen erste VDE-Normalien für 
Aluminiumwicklungen. Es benötigte umfangreiche Forschungsarbeiten, Erfahrungen und Beobachtungen im Betrieb sowie (kriegswirtschaftliche) Kosten-Nutzen-Rechnungen, um das beste Wicklungsmaterial für jede Motorenart herauszufinden. Am wirtschaftlich günstigsten erschien es schließlich, so Georg Dettmar 1916, „dass die Wicklungen von Drehstrommotoren unter 0,3 Kilowatt ganz aus Kupfer, von 0,8 bis 10 Kilowatt ganz aus Aluminium, von 10 bis 80 Kilowatt ganz aus Zink und darüber hinaus ganz aus Aluminium gebaut werden“ (Dettmar 1916: $561 \mathrm{f}$.).

Ein weiteres Indiz dafür, wie stark die maschinelle Qualität durch Verwendung von Ersatzstoffen während des Ersten Weltkriegs sank, lieferten die Firmen selbst, so Dettmar weiter. Alle Unternehmen wollten bei Maschinen und Apparaten „welche nur zum geringen Teil aus Ersatzmaterial hergestellt sind, jetzt überhaupt keine Garantieverpflichtungen übernehmen." (Ebd.: 574). Dieser Zustand wurde von den Abnehmern, die aus Friedenszeiten derartige Garantien gewohnt waren, logischerweise als unbefriedigend empfunden. VDE-Mitglieder drängten deshalb die Industrie dazu, wenigstens partiell Garantien zu übernehmen.

Selbst Universitätsprofessoren, deren stoffanalytische Arbeiten als überaus wichtig für die Ersatzstoffforschung angesehen wurden, litten unter den unzulänglichen Materialqualitäten. So bat Wilhelm Borchers, Ordinarius für Metallhüttenkunde an der Technischen Hochschule Aachen, den preußischen Wissenschaftsminister Friedrich Schmidt-Ott darum, ihn von der Verpflichtung $\mathrm{zu}$ entbinden, in seinem Labor Stromleiter aus Ersatzstoffen zu verwenden. Stattdessen möge Schmidt-Ott ihm gestatten, von der Industrie erhaltene geldwerte Leistungen mit dem Ministerium zu verrechnen, um nach dem Krieg in die Erneuerung der elektrischen Leitungen seines Institutes investieren zu können. Deren Material, „welches während der Kriegszeit aus billigeren Ersatzmetallen hergestellt werden muss," sei aufgrund der damit verbundenen Stromersparnisse „durch besser leitendes Material (insbesondere Kupfer) “ zu ersetzen. $^{10}$

\section{Leichtbau als Werkstoff-Sparstrategie}

Einen wichtigen Anteil an der Bewältigung von Materialversorgungsengpässen hatten konstruktive Strategien und fertigungstechnische Kniffe. Produkte wurden so neu konstruiert, dass die kritischen Bauteile aus Sparstoffen weggelassen oder verkleinert werden konnten. Diese Strategie des Werkstoffsparens ging vielfach Hand in Hand mit der ohnehin allgemein angestrebten Absicht des Leichtbaus. Fritz Mayr, Mitglied des VDI und Mitarbeiter in der Gutehoffnungshütte AG, leitete seinen Beitrag zu Möglichkeiten der Werkstoffeinsparung bei Stütz- und Tragbauteilen im Maschinenbau mit den Worten ein, dass diese „nicht nur durch Werkstoffaustausch, sondern in vielen Fällen durch grundsätzliche Änderungen der konstruktiven Gestaltung erreicht" würde: 
Die erste Aufgabe des Ingenieurs ist, zu prüfen, ob nicht durch grundsätzliche [...] Maßnahmen eine Verringerung der den bisherigen Werkstoffaufwand bedingenden Kräfte erzielt werden kann [...]. Eine derartige Lösung bringt einen wirklichen technischen Forschritt und ist der eleganteste Weg zur Werkstoffersparnis. (Mayr 1942: 27)

Mayr stellte eine konstruktive Einsparmöglichkeit bei einem Schiffsdieselmotor vor, auf dessen Kurbelwelle bei traditioneller Kröpfung in waagrechter Richtung starke Verbiegungskräfte wirkten. Dadurch wurde eine zusätzliche Versteifung des Maschinenfundamentes erforderlich (ebd.: 27-29). Eine Versetzung der Kröpfungsabfolge, so Mayr, verringerte hingegen die Zug-, Druck und Verbiegungskräfte. Deshalb könne bei einer derartigen Ausführung auf eine Aussteifung des Maschinenfundamentes verzichtet werden. Mit dieser Argumentation stellte Mayr anhand einer typischen Leichtbaukonstruktion den Nebeneffekt des Einsparens von Werkstoffen als Haupteffekt dar (vgl. Abb. 2). Eine vergleichbare Argumentation wurde bei elektrisch betriebenen Pumpen verwendet.

\section{Pumpen}

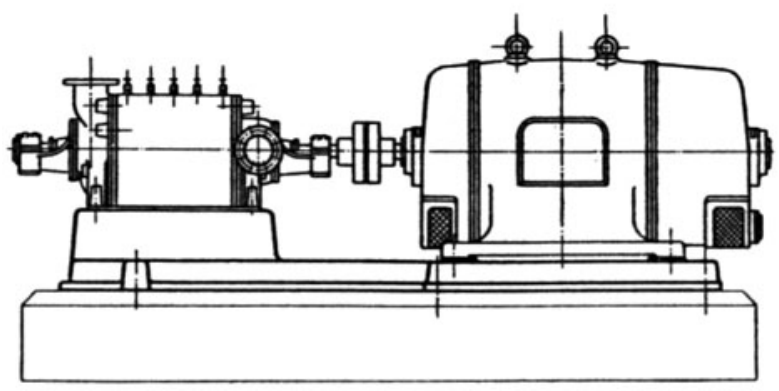

Gemeinsame Grundplatte für Pumpe und Motor einer $400 \mathrm{~kW}$ Wasserhaltungskreiselpumpe. Gewicht: $1600 \mathrm{~kg}$

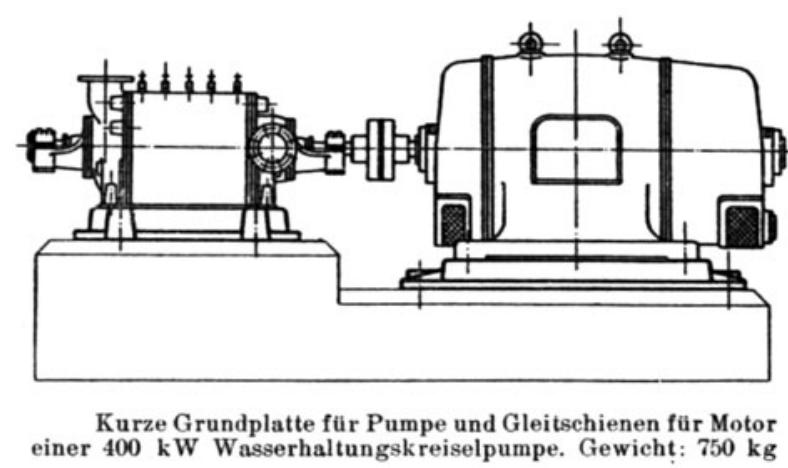

Abb. 2 Gusseiserne Maschinen-Grundplatten und -ständer eines Elektromotors mit Kreiselpumpe werden durch geschweißte Bleche und Betonfundamente ersetzt. (Verein Deutscher Ingenieure 1940: 48, Bild 3 und 4) 
Leichtbaukonstruktionen waren vor allem seit den 1920er Jahren weltweit Gegenstand der Konstruktionstechnik. Sie hatten ihre Wurzeln im verbesserten Wirkungsgrad, geringeren Betriebsstoffverbrauch, optimierten Maschinenlaufeigenschaften und in der geringeren Wartungsintensität. Die bis dahin eher als Nebeneffekt betrachtete Werkstoffersparnis wurde nun, da politisch opportun, ins Zentrum gerückt. Ein ähnliches Argumentationsmuster lässt sich bei der Herstellung von Eisenbahnwaggons beobachten. Die aus mehreren Teilen bestehenden, geschweißten Rahmenkonstruktionen waren leichter als die älteren genieteten. Obwohl bereits in der Zwischenkriegszeit ein Thema und zum Zeitpunkt der Veröffentlichung 1942 nicht mehr neu, wurde diese Tatsache als Werkstoff sparende Neuheit dargestellt (Hilpert 1928) Ähnlich stilisierten manche Ingenieure während der NS-Autarkiepolitik das damals bereits international übliche Schweißen zum Werkstoffsparverfahren hoch (Burckhardt 1937).

\section{Werkstoffgerechte Konstruktion}

Während der Zeit des Nationalsozialismus wurde das Stoffsparen in projektbezogene wissenschaftliche Arbeitsgemeinschaften von vornherein integriert. Bei manchen Ingenieuren und Konstrukteuren standen Arbeiten zu Austauschstoffen jedoch explizit im Mittelpunkt ihres Schaffens, wie etwa bei Hugo Wögerbauer, Konstrukteur bei Siemens und Professor für Feinwerktechnik an der Technischen Universität München. Er war als „Sparkommissar“ für den Wehrbezirk III Berlin zuständig und später für den Reichsminister für Bewaffnung und Munition tätig (Hellige 1995: 146-157, Wögerbauer 1940b). Ab 1940 gab er eine Schriftenreihe im VDI-Verlag unter dem Titel Werkstoffsparen heraus (Wögerbauer 1940a). Wögerbauer formulierte 1943 mit seinem Buch Werkstoffsparen in Konstruktion und Fertigung den ersten Entwurf für eine Systematisierung und Rationalisierung von Konstruktion und Werkstoffeinsatz (Hellige 1995: 146-157). Bereits das vorangestellte Motto des Bandes bezeugte das Dilemma, in dem sich die deutsche Industrie mit ihrem gleichzeitigen Mangel an Werkstoffen und Konstrukteuren befand: „In dem Maße, in welchem bei einem Erzeugnis der Werkstoffaufwand verringert wird, wächst der für die Konstruktion nötige Anteil geistiger Arbeit."(Wögerbauer 1940a: Vorbemerkung).

Es sei nicht damit getan, so Wögerbauer 1940, Werkstoffe einfach einzusparen oder zu ersetzen. Vielmehr müsse das Thema systematisch und analytisch aufgearbeitet werden: „Werkstoffumstellung heißt, nicht nur bei vorhandenen Konstruktionen den Werkstoff ändern, sondern auch neue Konstruktionen mit anderen Werkstoffen schaffen, als sie früher für einen ähnlichen Zweck richtig gewesen wären." (Wögerbauer 1940c: 4) Später, in seinem Werk Werkstoffsparen, fasste er 23 Gebrauchseigenschaften und fünf 
Verarbeitungseigenschaften von Werkstoffen zusammen (Wögerbauer 1940a: 27). In seinem 1943 publizierten Buch Die Technik des Konstruierens rückte er das Thema der Werkstoffwahl in einen noch größeren Zusammenhang. Darin stellte er 18 Betriebsaufgaben 23 Verwirklichungsaufgaben gegenüber. Unter Betriebsaufgaben fasste er alle relevanten „Anforderungen des Kundenbetriebs“ zusammen und unter „Verwirklichungsaufgaben“ die Anforderungen, die von Naturgesetzen, Baustoff und Werkstoff ausgingen. Mit Hilfe von schematisierten Bildtafeln setzte er diese Größen unter verschiedenen Gesichtpunkten zueinander in Beziehung (Wögerbauer 1943: 82 f.).

Wögerbauer integrierte in seine Konstruktionslehre alle Aspekte des deutschen Werkstoffmanagements. Sein eigentliches Motiv bestand darin, „das Konstruieren [...] zu rationalisieren“. Ihm ging es darum, den „Konstruktionszeitaufwand“, die „Güte des Erzeugnisses“, die „Kosten der Herstellung“ und den „Aufwand an Sparstoff“ (auch als „Sparstoffinhalt" bezeichnet) zu optimieren (Wögerbauer 1943: 160). Er ging davon aus, „daß der volkswirtschaftlich richtige Einsatz der Baustoffe nur durch Beachtung gewisser konstruktiver Regeln in jeder Hinsicht einwandfrei erzielt werden kann" (ebd.: VII). Damit trug er den älteren Bemühungen um „werkstoffgerechte Konstruktion“ Rechnung, deren Ziele und Ergebnisse nunmehr von der NS-Werkstoffpolitik überformt wurden. ${ }^{11}$ Mit diesem analytischen und von reichen Erfahrungen geleiteten Blick kam der Sparstoffkommissar Wögerbauer zu einem differenzierten und mitunter sogar kritischen Bild der bisherigen deutschen Ersatzstoffforschung. Die Verwendung von Werkstoffen „mit geringstem Sparzwang [...] muß sehr sorgfältig auf ihre Erfüllbarkeit und Zweckmäßigkeit geprüft werden" (Wögerbauer 1940a: 30 f.). Nicht immer mache die Forcierung der Umstellung volkswirtschaftlich Sinn. Deshalb solle man vor dem Beginn der Lösung einer Konstruktionsaufgabe die von ihm angegebenen Kriterien durchdenken:

Durch Umstellung des kritischen Teiles von einem in einer bestimmten physikalisch-chemischen Eigenschaft hochwertigen Werkstoff mit geringerem Sparzwang ergibt sich oftmals größeres Volumen (größerer Wellendurchmesser, größerer Eisenquerschnitt). Das kritische Teil für sich allein wird durch die Umstellung volkswirtschaftlich günstiger geworden sein. Da aber zur Aufrechterhaltung der Wirkung des Gerätes die anderen Teile mit dem kritischen Teil vergrößert werden müssen, kann der ursprünglich verhältnismäßig niedrige Sparstoffgehalt der abhängigen Teile (Lager, Wicklung) durch die Volumenvergrößerung so ansteigen, daß für das Gesamtgerät nicht nur keine Ersparnis, sondern sogar ein erhöhter Sparstoffaufwand eintritt (Wögerbauer 1940a: 30 f.).

\section{Zusammenfassende Ergebnisse: Ersatzstoffforschung als technische Praxis}

Die Ersatzstoffforschung gehörte im betrachteten Zeitraum nicht zum Gegenstandbereich der Naturwissenschaften und der Materialwissenschaft. 
Vielmehr zählte sie zum Aufgabenkreis der Werkstofftechnik und insbesondere der Konstruktionslehre und Konstruktionspraxis. Nicht die „Züchtung“ eines bestimmten Werkstoffes sondern der Nachweis der Praxistauglichkeit eines Werkstoffs für einen bestimmten technischen Zweck war zu entwickeln. Dabei griffen Ingenieure und Konstrukteure auf eine Menge unterschiedlicher Wissensquellen zurück, physikalisch-chemische, metallkundliche sowie materialwissenschaftliche Forschungsergebnisse waren nur einige von vielen.

Das Konzept der Ersatz- beziehungsweise Austauschstoffe in den beiden Phasen relativer Autarkie hatte durchaus Erfolge zu verzeichnen. In welchem Umfang der Austausch gelang, lässt sich allerdings schwer beziffern. Es ist aber davon auszugehen, dass er im kriegswirtschaftlichen Rahmen einen nennenswerten Umfang erreichte und mit dazu beitrug, den Krieg zu verlängern. Auch bestand ein dichtes organisatorisches Netz von institutionellen Beziehungen zwischen Rüstungsstellen und Wirtschaftsstellen, etwa in Form von Arbeitsgemeinschaften staatlicher und privater metallkundlicher Forschung, die bemerkenswerte Ergebnisse hervorbrachten. Die vorliegende Arbeit lenkte den Blick stärker auf das Stoffmanagement in der Technik. Dadurch wurden die bestehenden Unterschiede zwischen materialwissenschaftlicher und werkstofftechnischer Forschung einerseits und einer durch Gebrauchswertforschung aufgeklärten konstruktiv-technischen Forschung andererseits deutlich. Die Beiträge von Konstrukteuren, Betriebsingenieuren und Werkstattmitarbeitern waren von essentieller Bedeutung. Sie waren es, die am konkreten Objekt Material einzusparen hatten, und sie waren es auch, die entscheiden mussten, an welchen Maschinenteilen neue Stoffe am wirtschaftlichsten und am betriebssichersten einzusetzen seien, und nur sie konnten nach einiger Zeit beurteilen, ob diese Entscheidung richtig gewesen war oder der Korrektur bedurfte. Die dafür eingesetzten Kräfte standen für andere Sektoren der Rüstungsforschung nicht weiter zu Verfügung, was ganz im Sinne der alliierten Blockadepolitik war.

In der ersten Hälfte des 20. Jahrhunderts war eine ganze Reihe von Metallen und Legierungen im Gebrauch, die jedoch noch nicht physikalisch und chemisch analysiert waren. Dies behinderte die Ersatzstoffforschung zunächst gar nicht. Denn die Ingenieure, die sich mit diesen Fragen befassten, sahen Forschungsbedarf vor allem auf dem Prüfstand sowie im praktischen Dauerbetrieb und weniger im metallographischen Labor. Bevor ein bestimmter Werkstoff eingesetzt wurde, mussten die Techniker dessen Form, Funktion und Belastungen kennen. Dies war nur möglich, wenn man die Beanspruchung ganzer technischer Systeme kannte. Ersatzstoffforschung bestand darin, spezifische Beanspruchungen von Maschinenbauteilen herauszufinden und danach aus altbekannten oder neuen Materialien jene auszuwählen, die am ehesten mit den kriegsökonomischen Bestimmungen vereinbar waren. Eine weitere Möglichkeit bestand darin, die Konstruktion so abzuändern, dass nur ein Minimum an Sparstoffen verbaut werden musste. 
Sowohl in Friedens- wie in Kriegszeiten rangierten damit technische und ökonomische Fragen vor den naturwissenschaftlichen. Ein wesentlicher Teil der werkstofftechnischen Forschungen zwischen 1914 und 1919 sowie zwischen 1933 und 1945 unterschied sich deutlich von der Forschung anderer Zeitspannen. Während zu Friedenszeiten betriebswirtschaftliche Kriterien im Zentrum standen, dominierten in den Kriegsökonomien und im NS-Vierjahresplan volkswirtschaftliche Kriterien. Daraus erwuchsen Anforderungen, die für die Ingenieure neuartig waren und in die sie sich erst einarbeiten mussten.

Die Durchsetzung des volkswirtschaftlichen Paradigmas in der materialwissenschaftlichen und werkstofftechnischen Forschung der Autarkieperioden betrieben staatliche Behörden mit Hilfe von Gesetzen und durch die Indienstnahme von technisch-wissenschaftlichen Vereinen und Wirtschaftsverbänden. Technisch-wissenschaftliche Vereine wie VDI, VDE und VDMA stellten bei der Organisation der Ersatzstoffforschung ein wesentliches Bindeglied zwischen staatlichen Stellen und Industrie dar. Die deutsche Industrie hatte zwar zunehmend Interesse daran, neue Werkstoffe, etwa für leistungsfähigere Aggregate zu schaffen. Demgegenüber war jedoch ihr betriebswirtschaftliches Interesse, vor 1914 beziehungsweise 1933 in Ersatzstoffe und ihre Forschung zu investieren nur gering ausgeprägt. Erst Not und Zwang, Patriotismus, Gesetzestreue sowie politische Überzeugung führten zum Umdenken.

Ersatzstoffforschung umfasste eine Vielzahl wissenschaftlicher und technischer Maßnahmen. Im Mittelpunkt stand das Ziel blockierte, beschlagnahmte und staatlich zwangsverwaltete Rohstoffe einzusparen. Eigentlich wäre der Begriff „Sparstoffforschung“ treffender gewesen, denn darum gruppierte sich eine Reihe wirtschaftlicher, technischer und wissenschaftlicher Forschungsanstrengungen, von denen die Substitution eines knappen Stoffs durch einen weniger knappen nur eine von vielen Maßnahmen darstellte. Die zentralen Probleme beim Übergang von eingeführten $\mathrm{zu}$ inländischen Werkstoffen zeigten sich im Betrieb. Sie mussten in den Fabrikhallen der metallschaffenden und metallverarbeitenden Industrie, aber in noch größerem Umfang bei den Herstellern von Fertigprodukten gelöst werden. Oft bot der Stoffmangel den Anlass dafür, sich überhaupt erstmals mit Werkstofffragen in ökonomischer Perspektive auseinanderzusetzen. Dem entsprechend noch schwieriger zu beurteilen waren die Eigenschaften der neuen Materialien während der Autarkieperioden. Die Ingenieure und die Industrie waren nicht nur von der Dringlichkeit, sondern auch von der Komplexität der Aufgabenstellung überrascht und teilweise überfordert.

Die große Bandbreite von Anlagen, Maschinen und Apparaten sowie deren Vielzahl an Typen und Ausführungen zwangen zu jeweils detaillierten und fallbezogenen Untersuchungen. Die Ergebnisse dieser Untersuchungen verzögerten 
bis Anfang der 1940er Jahre allgemeine Überlegungen zur Ersatzstoffforschung. Viele Ersatzstoffe waren aufwändiger zu bearbeiten und machten betriebstechnische Umstellungen notwendig. Oft zog eine Umstellung zwingend andere Umstellungen nach sich, da bestimmte Werkstoffpaarungen sich nicht vertrugen. Eine Werkstoffumstellung bedingte vielfach auch einen Mehraufwand und/ oder eine Umstellung der Betriebsstoffe, an denen jedoch ebenfalls häufig Mangel herrschte.

Diese Zwangslage und die daraus resultierenden, sich oft multiplizierenden Schwierigkeiten bewirkten, dass auf gewohnte Produktqualität verzichtet und zur Improvisation übergegangen wurde. Sicherheitsstandards wurden gesenkt, Wirkungsgrade verringert und Lebensdauerspannen herabgesetzt. Obwohl die Ingenieure angetreten waren, durch volkswirtschaftliche Rationalisierung alle Kräfte für den Krieg zu optimieren, mussten sie eine Minderleistung der Produktionsmittel in Kauf nehmen. Häufig bestand der einzige Ausweg aus diesem Dilemma darin, technische Teilkomponenten neu zu dimensionieren und konstruktiv zu verändern. Dies bedingte in aller Regel eine Neukonstruktion ganzer Aggregate, für die die Kriegswirtschaften jedoch keine Ressourcen bereitstellen konnten.

Die Ingenieure sahen in diesem Aufgabenkomplex aber auch eine Chance zur Profilbildung gegenüber politischen Instanzen. Das Werkstoffsparen wurde immer stärker als Bestandteil der Konstruktionstätigkeit betrachtet und propagiert. Daher rückten viele Ingenieure die ursprünglichen Ziele des Leichtbaus (besserer Wirkungsgrad, geringere Wartungsintensität, Gewichtsund Betriebsstoffersparnis) in den Hintergrund und schoben stattdessen in der Zeit des Nationalsozialismus dessen Effekt der Werkstoffeinsparung in den Vordergrund. Dementsprechend entstanden ab 1940 in staatlichem Auftrag die ersten systematisch und generalisierend angelegten Darstellungen des Zusammenhangs zwischen Werkstoffökonomie, sparsamem Betrieb und optimaler Konstruktion.

\section{Anmerkungen}

1 Unter dem Begriff der Ersatzstoffforschung wird daher auch „Sparstoffforschung“ subsumiert. Dies schließt natürlich nicht aus, dass in einzelnen Fällen Ersatzstoffe auch in offenen Marktwirtschaften erfolgreich eingesetzt wurden, sofern sie einen signifikanten betriebswirtschaftlichen Vorteil bedeuteten.

2 Der Chemiker Fritz Haber versprach zu Beginn des Ersten Weltkriegs den deutschen Militärs die rasche Herstellung von Sprengstoffen auf Basis der von ihm und Carl Bosch entwickelten Ammoniaksynthese.

3 Freigabeanträge waren nur dann an die Metallfreigabestelle zu richten, wenn der entsprechende Industriezweig noch über keine eigene Beratungs- und Verteilungsstelle verfügte. Dies wird als Indikator dafür gewertet, dass zuerst der Staat einsprang und die Industrie erst danach motiviert werden konnte. 
4 Georg von Hanffstengel: Beiträge zur Frage des Sparstoffersatzes insbesondere beim Bau und Betrieb von Maschinen und Apparaten. Vortrag gehalten auf der außerordentlichen 38. Hauptversammlung des Vereins zur Wahrung der Interessen der chemischen Industrie Deutschlands e.V. am 19. Dezember 1916: 1, Bundesanstalt für Materialforschung und -prüfung, unverz. Bestand, Herdegen-Ordner beschriftet mit „Reibung und Verschleiß".

5 Georg Hanffstengel hatte in der Zeit der Weimarer Republik die Technisch-Wissenschaftliche Lehrmittelzentrale mit aufgebaut.

6 Siehe die Auflistung der erschienenen „Drucksachen“ und „Berichte“ der MetallBeratungs- und Verteilungsstelle für den Maschinenbau in: Betriebsergebnisse mit Calcium-Lagermetall (=Bericht M 224 der Metall-Beratungs- und Verteilungsstelle für den Maschinenbau), Charlottenburg 2, den 20. Dezember 1916: 6. Die meisten der Schriften sind als graue Literatur nicht an Bibliotheken nachgewiesen.

7 Tätigkeitsbericht, Staatliche Akademie für Technik Chemnitz an den Wirtschaftsminister in Dresden, 7. Oktober 1935, Universitätsarchiv Chemnitz, GdE 593, Bl. 77-78R, hier Bl. 78.

8 Georg von Hanffstengel: Beiträge zur Frage des Sparstoffersatzes insbesondere beim Bau und Betrieb von Maschinen und Apparaten. Vortrag gehalten auf der außerordentlichen 38. Hauptversammlung des Vereins zur Wahrung der Interessen der chemischen Industrie Deutschlands e.V. am 19. Dezember 1916: 1, Bundesanstalt für Materialforschung und -prüfung, unverz. Bestand, Herdegen-Ordner beschriftet mit „Reibung und Verschleiß".

9 Ebd.

10 W. Borchers an Unterrichtsminister Berlin, undat., Vermerk betr. Schreiben vom 25. Juli 1916 und 10. August 1916, Institutsakten Metallhüttenkunde, Hochschularchiv TH Aachen HAAc 924, nicht pag.

11 Ein Forschungsdesiderat besteht darin zu untersuchen, wie stark das Paradigma der werkstoffgerechten Konstruktion in Deutschland von der NS-Autarkiepolitik überformt wurde. Siehe Püttner 1939: 11-16.

\section{Literatur}

Adler, Dr. Reg. Rat, 1939, Roh und Werkstoffwirtschaft. Der Vierjahresplan: Zeitschrift für nationalsozialistische Wirtschaftspolitik, amtliche Mitteilungen des Beauftragten für den Vierjahresplan Ministerpräsident Reichsmarschall Herman Göring, 3, 882-883.

Anonym, 1915. Ersatz für einige im Interesse der Landesverteidigung beschlagnahmten Rohstoffe, Teil 6, Zeitschrift des Vereines Deutscher Ingenieure, 58, 545-550.

Anonym 2006. Kampf um Rohstoffe. Die knappen Schätze der Erde. Spiegel extra 5/2006.

Altmann, F.G., 1940. Austauschwerkstoffe für Getrieberäder, 1940. In: Verein Deutscher Ingenieure, $\mathrm{Hg}$., Werkstoffumstellung im Maschinen- und Apparatebau. Auszüge aus Vorträgen der gleichnamigen Vortragsreihe, veranstaltet im Herbst 1940 vom Verein Deutscher Ingenieure im Nationalsozialistischen Bund Deutsche Technik. Berlin: VDI-Verlag, 29-32.

Arbeitsgemeinschaft zur Förderung der Elektrowirtschaft, Hg., 1944. Merkblätter des Arbeitsausschusses für die Anwendung von Austauschstoffen und für Einsparung von NE-Metallen in elektrischen Anlagen. Ausgabe E für das Elektrofach. 5. Aufl. Berlin: Hugo L. Meyer.

Bauer, Heinrich-Wilhelm, 1939. Deutschlands Kolonialforderung und die Welt. Forderungen der deutschen Raum und Rohstoffnot. Leipzig: R. Bauer.

Buchheim, Christoph, 2002. "The ,Crisis before the Crisis'- The Export Engine out of Gear.' In: Harold James unter Mitarbeit von Elisabeth Müller-Luckner, Hg., The Interwar Depression in an International Context. München: Oldenbourg, 113-122.

Bundesverband der Deutschen Industrie, Hg., 2007. Anforderungen an Industrie und Politik. 2. BDI-Rohstoffkongress am 20. März 2007 in Berlin. Ergebnisbericht der BDI-Präsidialgruppe "Internationale Rohstofffragen“. Berlin: Industrie Förderung GmbH.

Bürgel, Heinrich, 1937. Deutsche Austausch-Werkstoffe. Berlin: Springer. 
Burchardt, Lothar, 1968. Friedenswirtschaft und Kriegsvorsorge. Deutschlands rüstungswirtschaftliche Bestrebungen vor 1914. Boppard: Boldt.

Burckhardt, Hermann, 1937. Werkstoff sparen durch Schweißen. Der Vierjahresplan: Zeitschrift für nationalsozialistische Wirtschaftspolitik, amtliche Mitteilungen des Beauftragten für den Vierjahresplan Ministerpräsident Reichsmarschall Herman Göring, 1, 345-348.

Dettmar, Georg, 1916. Der Ersatz von Sparstoffen in der Elektrotechnik. Elektrotechnische Zeitschrift, 1:561-565.

Eberhardt, Eugen/Kolbe, Walther, 1938. Die Anordnungen der Überwachungsstelle für unedle Metalle. Berlin: Deutscher Verlag für Politik und Wirtschaft.

Flachowsky, Sören, 2008. Von der Notgemeinschaft zum Reichsforschungsrat. Wissenschaftspolitik im Kontext von Autarkie, Aufrüstung und Krieg. Stuttgart: Steiner.

Frech, Albert, 1847. Theoretisch praktische Anleitung die Schiessbaumwolle und das Schiesspulver sowie andere Ersatzmittel derselben, nämlich explosive Hobelspäne, explosiven Flachs oder Hanf zu bereiten. Heilbronn: Landherr.

Grouven, Hubert, 1873. Ueber die Ersatzfrage des Peru-Guano. Leipzig: Schmidt.

Heidebroeck, E. 1940, Grundlagen, des betriebssicheren Lagerlaufs (Zu dem Vortrag Heidebroek). In: Verein Deutscher Ingenieure, Hg., Werkstoffumstellung im Maschinen- und Apparatebau. Auszüge aus Vorträgen der gleichnamigen Vortragsreihe, veranstaltet im Herbst 1940 vom Verein Deutscher Ingenieure im Nationalsozialistischen Bund Deutsche Technik. Berlin: VDIVerlag, Werkstoffumstellung: 8-11.

Hanffstengel, Georg von, 1916. Über den Ersatz der Sparstoffe im Maschinenbau. Beilage zum Bericht über die 483. Sitzung des Karlsruher Bezirksvereins des Vereins Deutscher Ingenieure am 5.4.1916, Karlsruhe. Ohne Seitenangabe.

Hanffstengel, Georg von/Bürgel, Heinrich, 1937. Vorwort und Einführung. In: Ders.. Deutsche Austausch-Werkstoffe. Berlin: Springer.

Hellige, Hans Dieter, 1995. Hierarchische Ablaufsteuerung oder kooperative Bewältigung von Problemzusammenhängen? Zur Geschichte von Modellen des Konstruktionsprozesses. In: Hellmut Lange und Wilfried Müller, Hg., Kooperation in der Arbeits- und Technikgestaltung. Münster/Hamburg: Lit: 135-164.

Herttrich, Hans, 1940. Die volkswirtschaftlichen und technischen Aufgaben des Metalleinsatzes. In: Verein Deutscher Ingenieure, Hg., Werkstoffumstellung im Maschinen- und Apparatebau. Auszüge aus Vorträgen der gleichnamigen Vortragsreihe, veranstaltet im Herbst 1940 vom Verein Deutscher Ingenieure im Nationalsozialistischen Bund Deutsche Technik. Berlin: VDIVerlag, Werkstoffumstellung, 1-4.

Hildebrand, Klaus, 1969. Vom Reich zum Weltreich. Hitler, NSDAP und koloniale Frage 1919-1945. München: Fink.

Hilpert, August, 1928, Werkstoffersparnis beim Schweißen. In: Verein Deutscher Eisenhüttenleute, Hg., Stahl und Eisen als Werkstoff. Bd. II. Düsseldorf: Verlag Stahleisen, 92-97.

Hoyer, Egbert von, 1887. Die Fabrikation des Papiers nebst Gewinnung der Fasern aus Ersatzstoffen. Braunschweig: Vieweg.

Kahn, Daniela, 2006. Die Steuerung der Wirtschaft durch Recht im nationalsozialistischen Deutschland. Das Beispiel der Reichsgruppe Industrie. Frankfurt a. Main: Klostermann.

Kammerer, Otto, 1917. Grundsätzliche Beobachtungen bei Lagerversuchen (= Bericht M 223 der „Metall-Beratungs- und Verteilungsstelle für den Maschinenbau), Drucksache des VDMA Charlottenburg 1917, Nr. 5.

van de Kerkhof, Stefanie, 2006. Von der Friedens- zur Kriegswirtschaft. Unternehmensstrategien der deutschen Eisen- und Stahlindustrie vom Kaiserreich bis zum Ende des Ersten Weltkrieges. Essen: Klartext.

Kessner, Arthur u. a, 1915. Rohstoffersatz. Berlin: VDI-Verlag.

Kessner, Arthur u. a., 1916. Rohstoffersatz. 2. Aufl. Berlin: VDI-Verlag.

Kessner, Arthur u. a. 1921. Ausnutzung und Veredlung deutscher Rohstoffe. (3. Aufl. von Rohstoffersatz), Berlin: VDI-Verlag.

Kessner, Arthur u.a. 1940. Werkstoffeinsparung und Werkstoffumstellung bei Kreiselpumpen. In: Verein Deutscher Ingenieure, Hg., 1940, Werkstoffumstellung im Maschinen- und Apparatebau. Auszüge aus Vorträgen der gleichnamigen Vortragsreihe, veranstaltet im Herbst 1940 vom Verein Deutscher Ingenieure im Nationalsozialistischen Bund Deutsche Technik. Berlin: VDI-Verlag, 45-48. 
Leiser, M., 1921. Ersatzmetalle in der allgemeinen Friedensindustrie. In: Verein Deutscher Ingenieure, Hg. [Arthur Kessner]. Ausnutzung und Veredlung deutscher Rohstoffe. (3. Aufl. von Rohstoffersatz), Berlin: VDI-Verlag, 168-191.

Lesser, Ian O., 1989. Resources and Strategy. New York: St. Martińs Press.

Ludwig, Karl-Heinz/König, Wolfgang, 1981. Technik, Ingenieure und Gesellschaft. Geschichte des Vereins Deutscher Ingenieure 1856-1981. Düsseldorf: VDI-Verlag.

Luxbacher, Günther, 2001. Die technologische Mobilisierung der Botanik. Konzept und Wirkung der Technischen Rohstofflehre und Warenkunde im 19.Jahrhundert. Technikgeschichte, 68, 307-333.

Luxbacher, Günther, 2004. Roh- und Werkstoffe für die Autarkie. Textilforschung in der KaiserWilhelm-Gesellschaft. In: Carola Sachse im Auftrag der Präsidentenkommission der MaxPlanck-Gesellschaft zur Förderung der Wissenschaften e.V, Hg., Forschungsprogramm „Geschichte der Kaiser-Wilhelm-Gesellschaft im Nationalsozialismus, Bd. 18. Selbstverlag.

Luxbacher, Günther, 2006. Wertarbeit mit Ersatzstoffen? Ausstellungen als Bühne deutscher Werkstoffpolitik 1916 bis 1942. Dresdner Beiträge zur Geschichte der Technikwissenschaften, 31, S. 3-24.

Luxbacher, Günther, 2007. Kohle-Öl-Benzin. Die Fischer-Tropsch-Synthese in der interinstitutionellen Kooperation. In: Helmut Maier, Hg., Gemeinschaftsforschung, Bevollmächtigte und der Wissenstransfer. Die Rolle der Kaiser-Wilhelm-Gesellschaft im System kriegsrelevanter Forschung des Nationalsozialismus. Göttingen: Wallstein, 453-502.

Luxbacher, Günther, 2010. Ersatzstoff, Sparstoff, Heimstoff. Metallforschung in Deutschland zwischen 1920 und 1970. In: Karin Orth und Willi Oberkrome, Hg., Die Deutsche Forschungsgemeinschaft 1920-1970. Forschungsförderung im Spannungsfeld von Wissenschaft und Politik. Göttingen: Wallstein, 163-181.

Mäkelt, Heinrich, 1921. Metallersatz bei chemischen Vorgängen. In: Verein Deutscher Ingenieure, Hg. [Arthur Kessner]. Ausnutzung und Veredlung deutscher Rohstoffe. (3. Aufl. von Rohstoffersatz), Berlin: VDI-Verlag, 191-215.

Maier, Helmut, 2002. Ideologie, Rüstung und Ressourcen. Das Kaiser-Wilhelm-Institut für Metallforschung und die „Deutschen Metalle“ 1933-1945. In: Ders. (Hg)., Rüstungsforschung im Nationalsozialismus. Organisation, Mobilisierung und Entgrenzung der Technikwissenschaften. Göttingen: Wallstein, 357-388.

Maier, Helmut, 2007. Forschung als Waffe. Rüstungsforschung in der Kaiser-Wilhelm.-Gesellschaft und das Kaiser-Wilhelm-Institut für Metallforschung. 1900-1945/48, 2 Bände. Göttingen: Wallstein.

Marsch, Ulrich 2000. Zwischen Wissenschaft und Wirtschaft. Industrieforschung in Deutschland und Großbritannien 1880-1936. Paderborn: Schöningh.

Mayr, Fritz, 1942. Werkstoffeinsparung bei Stütz- und Tragbauteilen im Maschinenbau. Konstruktive Maßnahmen und Werkstoffaustausch. In: Hermann Elsner, Hg., Konstruieren in neuen Werkstoffen. Berlin: VDI-Verlag, 27-29.

Müller, Alfred, 1955. Die Kriegsrohstoffbewirtschaftung 1914-1918 im Dienste des deutschen Monopolkapitals. Berlin: Akademie-Verlag.

Petzina, Dieter, 1968. Autarkiepolitik im Dritten Reich. Der nationalsozialistische Vierjahresplan. Stuttgart: DVA.

Pleich, Arpád, 1918. Die kriegswirtschaftliche Organisation Deutschlands (Geheim!), Wien: k.k. Hof- und Staatsdruckerei.

Püttner, H., 1939. Werkstoffgerechte Gestaltung von Maschinenteilen aus Leichtmetallguß (Aluminiumund Magnesiumlegierungen). Metallwirtschaft, Metallwissenschaft, Metalltechnik, 18, 11-16.

Radkau, Joachim, 1989. Technik in Deutschland. Vom 18. Jahrhundert bis zur Gegenwart. Frankfurt a. Main: Suhrkamp.

Rasch, Manfred, 1991. Wissenschaft und Militär. Die Kaiser Wilhelm Stiftung für kriegstechnische Wissenschaft. Militärgeschichtliche Mitteilungen, 44, 73-120.

Rathenau, Walther, 1916. Deutschlands Rohstoffversorgung. Berlin: S. Fischer.

Reinhardt, Carsten, 1997: Forschung in der chemischen Industrie. Die Entwicklung synthetischer Farbstoffe bei BASF und Hoechst 1863-1914. Freiberg: TU Bergakademie.

Reith, Reinhold, 1993. Vom Umgang mit Rohstoffen in historischer Perspektive. Rohstoffe und ihre Kosten als ökonomische und ökologische Determinanten der Technikentwicklung. Johann Beckmann-Journal, 7, 87-99.

Reith, Reinhold, 2001. Von der Materie zum Material. Werkstoffe in der Geschichte. Technikgeschichte, 68, 301-306. 
Rürup, Reinhard, Hg., 1979. Wissenschaft und Gesellschaft. Beiträge zur Geschichte der Technischen Universität Berlin 1879-1979. Bd. 1, Berlin u. a.: Springer, 278.

Schölzel, Christian, 2006: Walther Rathenau: eine Biographie. Paderborn: Schöningh.

Schulz, Matthias, 1997. Deutschland, der Völkerbund und die Frage der europäischen Wirtschaftsordnung 1925-1933. Hamburg: Krämer.

Schwarz, Roland, 1993. Aluminium für die Elektrotechnik. Wirtschaftliche und politische Bedingungen einer Werkstoffinnovation zu Beginn des 20. Jahrhunderts. Technikgeschichte, 60, 107-128.

Siegenthaler, Hansjörg, Hg., 1990. Ressourcenverknappung als Problem der Wirtschaftsgeschichte. Berlin u. a.: Dunker \& Humblot.

Smith, George Otis Hg., 1919. The Strategy of Minerals. A Study of the Mineral Factor in the World Position of America in War and in Peace. New York, London: D. Appleton and Company.

von Soden, Graf E./Ullrich, M. 1940. Bewährung der Austauschstähle EC-80, EC-100 und ECMo200 für Getrieberäder. In: Verein Deutscher Ingenieure, Hg., Werkstoffumstellung im Maschinen- und Apparatebau. Auszüge aus Vorträgen der gleichnamigen Vortragsreihe, veranstaltet im Herbst 1940 vom Verein Deutscher Ingenieure im Nationalsozialistischen Bund Deutsche Technik. Berlin: VDI-Verlag, 26-29.

Sombart, Werner, 1987. Der moderne Kapitalismus, Bd. 3,1. Berlin u. a.: Duncker \& Humblot, 70.

Szöllösi-Janze, Margit, 1998. Fritz Haber 1868-1934. Eine Biographie. München: Beck.

Teichert, Eckart, 1984. Autarkie und Großraumwirtschaft in Deutschland 1930-1939. Außenwirtschafspolitische Konzeptionen zwischen Wirtschaftskrise und Zweitem Weltkrieg. München: Oldenbourg.

Thum, August, 1944. Die Entwicklung von der Lehre von der Gestaltfestigkeit. In: Zeitschrift des Vereins Deutscher Ingenieure im Nationalsozialistischen Bund Deutscher Technik, Nr. 45/46, 609-615.

Uhde, Hans, 1941. August Thum zum 60. Geburtstag. Forschung auf dem Gebiete des Ingenieurwesens, 12, 161.

Voigt, Andreas, 1912. Technische Oekonomik. In: Leopold von Wiese, Hg., Wirtschaft und Recht der Gegenwart. Ein Leitfaden für Studierende der technischen Hochschulen und Bergakademie sowie der praktischen Techniker und Bergleute, Bd. 2, Tübingen: Mohr, 221-315.

Wengenroth, Ulrich, 2002. Die Flucht in den Käfig: Wissenschafts- und Innovationskultur in Deutschland 1900-1960. In: Rüdiger vom Bruch und Brigitte Kaderas, Hg., Wissenschaften und Wissenschaftspolitik. Bestandsaufnahmen zu Formationen, Brüchen und Kontinuitäten im Deutschland des 20. Jahrhunderts. Stuttgart: Steiner, 52-59.

Verein Deutscher Ingenieure, Hg., 1940, Werkstoffumstellung im Maschinen- und Apparatebau. Auszüge aus Vorträgen der gleichnamigen Vortragsreihe, veranstaltet im Herbst 1940 vom Verein Deutscher Ingenieure im Nationalsozialistischen Bund Deutsche Technik. Berlin: VDI-Verlag.

Wiedenfeld, Kurt, 1936. Die Organisation der Kriegsrohstoff-Bewirtschaftung im Weltkriege. Hamburg: Hanseatische Verlagsanstalt.

Wögerbauer, Hugo, 1940a. Werkstoffsparen in Konstruktion und Fertigung [=Reihe Werkstoffsparen, Heft 1], Berlin: VDI-Verlag.

Wögerbauer, Hugo, 1940b. Werkstoffsparen auch in Zukunft, Rundschau Deutscher Technik. Wochenzeitung des Nationalsozialistischen Bundes Deutscher Technik, 20, 26. September 1940: 2 .

Wögerbauer, Hugo, 1940c. Angriffspunkte und Verantwortliche bei der Werkstoffumstellung. In: Verein Deutscher Ingenieure, Hg., Werkstoffumstellung im Maschinen- und Apparatebau. Auszüge aus Vorträgen der gleichnamigen Vortragsreihe, veranstaltet im Herbst 1940 vom Verein Deutscher Ingenieure im Nationalsozialistischen Bund Deutsche Technik. Berlin: VDI-Verlag, 4-7.

Wögerbauer, Hugo, 1943. Die Technik des Konstruierens. 2. Aufl. München: Oldenbourg.

Wronkow, J., 1921. Ersatzmetalle in der Elektrotechnik. In: Verein Deutscher Ingenieure, Hg. [Arthur Kessner]. Ausnutzung und Veredlung deutscher Rohstoffe. (3. Aufl. von Rohstoffersatz), Berlin: VDI-Verlag, 168-191.

Zachmann Karin, 1995. Wirkungsgrad contra Wertgrad. Zur Entstehung des Konflikts zwischen der technischen und der ökonomischen Auffassung vom Wirtschaften. Technikgeschichte, 62, 103-131. 
Günther Luxbacher

Institut für Philosophie, Literatur-,

Wissenschafts- und Technikgeschichte

Abt. Technikgeschichte

Technische Universität Berlin

Franklinstraße 28-29

10587 Berlin

Deutschland

E-Mail: guenther.luxbacher@tu-berlin.de 